\title{
Radio over Fiber: An Alternative Broadband Network Technology for IoT
}

\author{
Diego F. Paredes-Páliz ${ }^{1}$, Guillermo Royo ${ }^{1}$, Francisco Aznar², Concepción Aldea ${ }^{1}$, \\ Santiago Celma ${ }^{1}$ \\ ${ }^{1}$ Electronic Design Group (GDE) - Instituto de Investigación en Ingeniería de Aragón (I3A) \\ Universidad de Zaragoza, Pedro Cerbuna 12, 50009, Zaragoza, Spain. \\ Tel.+34-876553547, e-mail: dparedes@unizar.es \\ 2 Electronic Design Group - Instituto de Investigación en Ingeniería de Aragón (I3A) \\ Centro Universitario de la Defensa, Academia General Militar. Ctra. Huesca s/n., 50090, Zaragoza, Spain
}

\begin{abstract}
Wireless broadband access networks have been positioning themselves as a good solution for manufacturers and users of IoT (internet of things) devices, due mainly to the high data transfer rate required over terminal devices without restriction of information format. In this work, a review of two Radio over Fiber strategies is presented. Both have excellent performance and even offer the possibility to extend wireless area coverage where mobile networks do not reach, or the 802.11 network presents issues. Radio Frequency over Fiber (RFoF) and intermediate Frequency over Fiber (IFoF) are two transmission strategies compatible with the required new broadband services and both play a key role in the design of the next generation integrated optical-wireless networks, such as 5G and Satcom networks, including on RAU (Remote Antenna Unit) new functionalities to improve their physical dimensions, employing a microelectronic layout over nanometric technologies.
\end{abstract}

\section{Technological Approach}

In new wireless access networks, there is an option to avoid the attenuation issue through distributed antenna systems (DAS) implementation. That is, a high-density network composed of small base stations (small cells) distributed by urban areas or industrial environments to provide good coverage over a specific area, as is shown in Figure 1. These cells, operating at millimeter waves, will need antennas of reduced size compared to current ones, which will simplify their deployment. On the other hand, reduced antenna size brings up the possibility that future base stations will be able to operate using massive multiple input-multiple output (MIMO) configurations to strongly increase the maximum data capacity, if individual channels are multiplexed over the same communications infrastructure.
A new telecommunications scenario is still being set, with fewer doubts that over most commonly used standards for IoT implementation (WiFi, Bluetooth, ZigBee, Thread, SigFox, NFC, etc.), the WiFi standard is the preferred option and has a greater development potential. In fact, there are many predictions that coincide in pointing out that the role played by theWiFi standard in IoT systems, relevant already, will become more important with time and will establish as the dominant option in the midterm.(Lopez-Perez et al. 2019)

\section{Wireless Broadband Integration}

In the last years, mixed fiber-wireless communication DAS fed by multi-mode fibers (MMF) are gaining space as the most promising solution to achieve efficient, cost-effective, and highcapacity transmissions in short range communications. (Lim et al. 2019)

These systems are flexible and there is a good compromise between data transmission capacity, accessibility, and the overall cost of installation and maintenance, allowing a good convergence of optical fiber capacity and wireless access flexibility. In this approach, the signal is generated and processed in a base station (BS) and is distributed through MMF to several remote antenna units (RAU), which provide an optical-wireless interface as is shown in Figure 2.(Lim et al. 2010)

\section{RAU Operation Scheme}

A dense RAU devices deployment means that the key to a DAS is to employ cost-effective components in the three main elements of the communication system. This is achieved with the use of MMF (Multimode fiber), which offers electromagnetic isolation and a large capacity, along with costeffective photonic devices, such as vertical-cavity surface-emitting lasers (VCSEL). 
Lastly, a low-cost, low-power, fully integrated RAU must be designed in cost-effective technologies such as CMOS and a moderate complexity to reduce the cost of DAS. An operation scheme of an electrical/optical (EO) and optical/electrical (OE) RAU domain converter is presented in Figure 3, with a description of main blocks and key parameters.

\section{RAU Architectures}

\section{Baseband over Fiber}

The BBoF scheme follows the typical configuration of optical communication systems for long-reach applications. With this scheme, very high bit rates in the order of several Gbps can be achieved. However, this scheme requires complex RAU configuration and design, as it must perform data modulation and demodulation, as well as frequency conversion, also increasing power consumption.

\section{Radio Frequency over Fiber}

On the opposite side, the RFoF scheme, called also in some scientific literature RoF, presents the simplest RAU architecture, because the RAU only has to perform opto/electrical (O/E) and electro/optical (E/O) conversion and signal amplification. It is the most flexible RAU, since as it works in the RF domain, it is transparent to the data encoding or communication standard.

\section{Intermediate Frequency over Fiber}

Halfway between $\mathrm{BBoF}$ and RFoF we find IFoF. These systems present advantages over both $\mathrm{BBoF}$ and RFoF. As in the RFoF approach, RAUs for IFoF do not require the implementation of $a$ modulator/demodulator. Therefore, the complexity

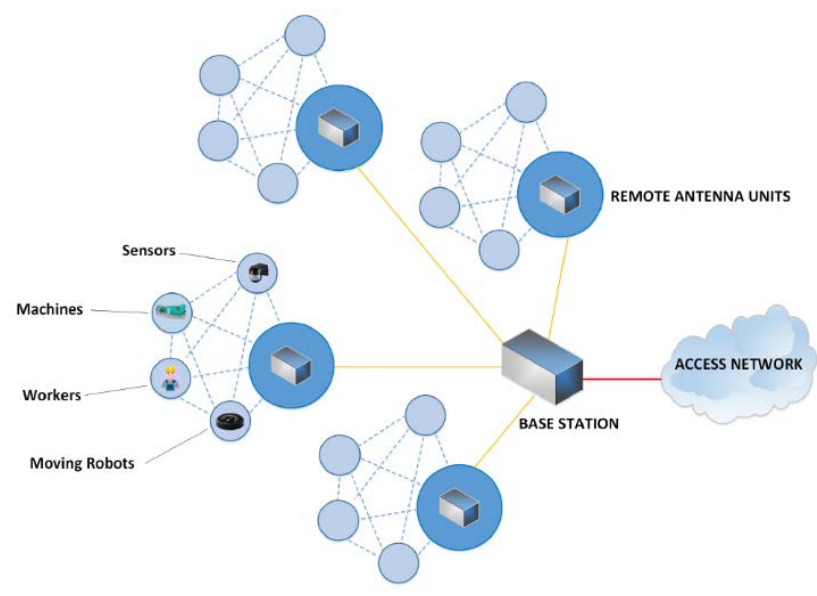

Fig. 1. Scheme of a distributed antenna system in the design of the RAU is much simpler than that in $\mathrm{BBoF}$ systems, and the power consumption is significantly lower.

\section{Conclusiones}

IFoF technology is the most promising solution for IoT network deployment due to workable electronic IC design and integration with currently available WiFi 802.11 and emerging WiFi 802.11ad/ay standards at $60 \mathrm{GHz}$. This can be the start point for future research to achieve a good RAU design and fabrication, that allow to measure the main parameters, to achieve good results employing monolithic technologies.(Paredes-Páliz et al. 2020)

\section{REFERENCIAS}

[1]. LIM, C., NIRMALATHAS, A., BAKAUL, M., GAMAGE, P., LEE, K.L., YANG, Y., NOVAK, D. and WATERHOUSE, R., 2010. Fiber-wireless networks and subsystem technologies. Journal of Lightwave Technology, vol. 28, no. 4, pp. 390-405. ISSN 07338724. DOI 10.1109/JLT.2009.2031423.

[2]. LIM, C., TIAN, Y., RANAWEERA, C., NIRMALATHAS, T.A., WONG, E. and LEE, K.L., 2019. Evolution of Radio-Over-Fiber Technology. Journal of Lightwave Technology, vol. 37, no. 6, pp. 1647-1656. ISSN 07338724. DOI 10.1109/JLT.2018.2876722.

[3]. LOPEZ-PEREZ, D., GARCIA-RODRIGUEZ, A., GALATI-GIORDANO, L., KASSLIN, M. and DOPPLER, K., 2019. IEEE 802.11be Extremely High Throughput: The Next Generation of Wi-Fi Technology beyond 802.11ax. IEEE Communications Magazine, vol. 57, no. 9, pp. 113-119. ISSN 15581896. DOI 10.1109/MCOM.001.1900338.

[4]. PAREDES-PÁLIZ, D.F., ROYO, G., AZNAR, F., ALDEA, C. and CELMA, S., 2020. Radio over Fiber: An Alternative Broadband Network Technology for IoT. Electronics [en línea], vol. 9, no. 11, pp. 1-8. ISSN 20799292. DOI 10.3390/electronics9111785. Disponible en: https://doi.org/10.3390/electronics9111785.
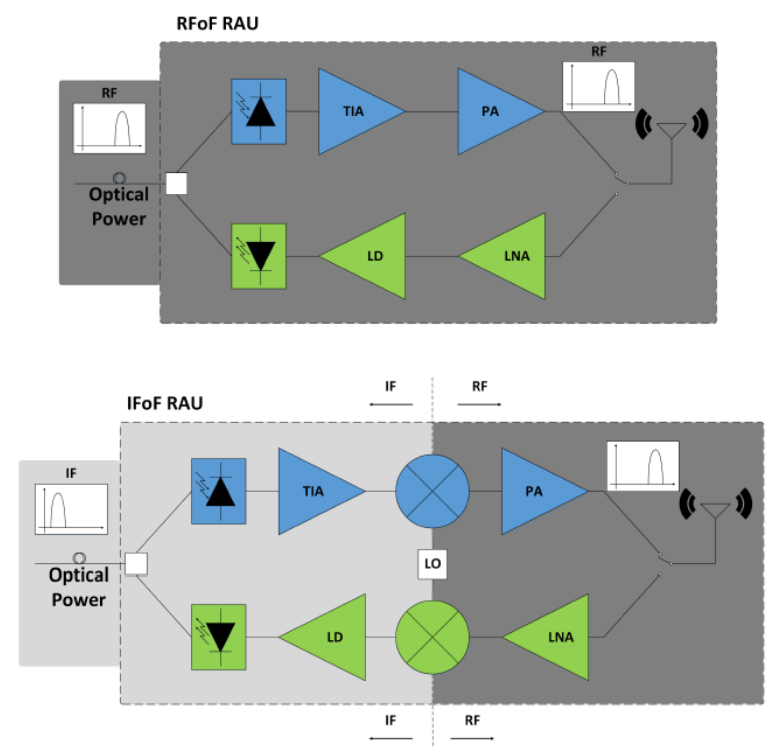

Fig. 2. RoF and IFoF architecture

Revista “Jornada de Jóvenes Investigadores del I3A”, vol. 8 (Actas de la IX Jornada de Jóvenes Investigadores del I3A -11 de diciembre de 2020). ISSN 2341-4790. 